\title{
Assessment of Observation Result
}

National Cancer Institute

\section{Source}

National Cancer Institute. Assessment of Observation Result. NCI Thesaurus. Code C70727.

A critical appraisal of the evidence of the association between an assessment and the results of an observation. 